\title{
Du Collège Manitou de La Macaza à l'Institution Kiuna d'Odanak : la genèse des établissements postsecondaires par et pour les Premières Nations au Québec
}

\section{EMANUELLE DUFOUR}

Volume 70, numéro 4, printemps 2017

URI : https://id.erudit.org/iderudit/1040572ar

DOI : https://doi.org/10.7202/1040572ar

Aller au sommaire du numéro

Éditeur(s)

Institut d'histoire de l'Amérique française

ISSN

0035-2357 (imprimé)

1492-1383 (numérique)

Découvrir la revue

Citer cet article

Dufour, E. (2017). Du Collège Manitou de La Macaza à l'Institution Kiuna d'Odanak : la genèse des établissements postsecondaires par et pour les Premières Nations au Québec. Revue d'histoire de l'Amérique française, 70(4), 5-33. https://doi.org/10.7202/1040572ar

\section{Résumé de l'article}

En 1973, dans les installations de l'ancienne base miliaire de La Macaza, au Québec, ouvrait l'un des premiers établissements postsecondaires destinés aux étudiants autochtones du Canada. Si l'expérience du Collège Manitou ne dura que quelques années, elle eut pourtant un effet considérable sur la fortification d'une identification collective, sur l'essor d'un leadership et sur le processus d'autonomisation autochtones au Canada et au Québec. À travers le recensement de sources primaires et secondaires et l'apport de nouveaux témoignages d'acteurs clés, cet article propose de retracer les grandes lignes qui ont caractérisé la création, le développement puis la fermeture du Collège Manitou.
Tous droits réservés Institut d'histoire de l'Amérique française, 2017

Ce document est protégé par la loi sur le droit d'auteur. L'utilisation des services d'Érudit (y compris la reproduction) est assujettie à sa politique d'utilisation que vous pouvez consulter en ligne.

https://apropos.erudit.org/fr/usagers/politique-dutilisation/ 


\title{
Du Collège Manitou
} de La Macaza à l'Institution Kiuna d'Odanak : la genèse des établissements postsecondaires par et pour les Premières Nations au Québec ${ }^{\mathrm{r}}$

\author{
EmANuelle Dufour \\ Department of Art Education \\ Université Concordia
}

RÉSUMÉ - En 1973, dans les installations de l'ancienne base miliaire de La Macaza, au Québec, ouvrait l'un des premiers établissements postsecondaires destinés aux étudiants autochtones du Canada. Si l'expérience du Collège Manitou ne dura que quelques années, elle eut pourtant un effet considérable sur la fortification d'une identification collective, sur l'essor d'un leadership et sur le processus d'autonomisation autochtones au Canada et au Québec. À travers le recensement de sources primaires et secondaires et l'apport de nouveaux témoignages d'acteurs clés, cet article propose de retracer les grandes lignes qui ont caractérisé la création, le développement puis la fermeture du Collège Manitou.

Abstract - Manitou College, one of the first Canadian post-secondary institutions for indigenous students, was created in 1973 on the abandoned site of the missile base located in La Macaza, Quebec. Despite its very short existence, Manitou College had a considerable impact on the definition of a collective indigenous identity and on the construction of First Nations leadership in Canada and Quebec. Using primary and secondary sources as well as new testimonies from key actors, this article aims to contextualize the rise and fall of Manitou College.

1. Je tiens à remercier Lise Bastien, Jacques Kurtness et Rémi Cadieux pour leurs précieux témoignages ainsi que tous les autres informateurs de la recherche. Je remercie également Sébastien BrodeurGirard, Marie-Pierre Bousquet, Martine Bourque, Bernard Hervieux, Michèle Vincelette, Thomas Poulette et Recherches amérindiennes au Québec pour leur collaboration. Je salue aussi le Conseil de recherche en sciences humaines du Canada (CRSH) et le Fonds de recherche du Québec - Société et culture (FRQSC) pour leur soutien. 
$\mathbf{E}^{n}$ n vertu de la Loi sur les Indiens de 1876 et de son amendement de $1920^{2}$, le premier pensionnat indien du Québec, St. Philip, est ouvert à Fort George en $1932^{3}$, soit plus d'un siècle après son homologue ontarien, le Mohawk Indian Residential School de Brantford. Au cours des années suivantes, cinq autres pensionnats ont été créés au Québec à l’intention d'enfants cris, innus, algonquins et atikamekw généralement âgés de 7 à 16 ans $^{4}$. Le régime des pensionnats autochtones fut mis en ouvre par le gouvernement fédéral et majoritairement administré au Québec par la congrégation des missionnaires oblats de Marie-Immaculée. Il visait essentiellement à éloigner les enfants autochtones de leurs familles et communautés dans le but d'empêcher la transmission de pratiques culturelles et sociales pouvant nuire au processus d'enculturation au sein de la société eurocanadienne et chrétienne $e^{5}$. Les études supérieures constituaient en soi une menace tangible pour le statut d'Indien des étudiants considérés comme présentant un "degré de civilisation» adéquat ${ }^{6}$. Les diplômés universitaires, ainsi que leurs conjoints et enfants, étaient émancipés ipso facto sous l'Acte pour amender et refondre les lois concernant les sauvages $^{7}$ de 1876 et de manière conditionnelle sous son amendement de $1880^{8}$. Ce n'est qu'avec le projet de loi C-31 de 1985 que les Autochtones émancipés et leurs descendants ont pu récupérer leur identité légale ${ }^{9}$. Bien que la laïcisation de l'éducation autochtone soit officiellement entrée en vigueur en 1969, le dernier pensionnat indien du Québec ne ferma ses portes qu’en 1980. Il faudra attendre l’année 1996 pour que se termine définitivement le régime des pensionnats indiens au Canada ${ }^{10}$.

2. An Act to Amend the Indian Act, S.C. 1919-20, c. 50 (10-11 Geo. V).

3. Le pensionnat anglican ne sera financé par le gouvernement fédéral qu'à partir de 1934. Commission de vérité et réconciliation du Canada, Pensionnats du Canada; L'histoire, partie 1, des origines à 1939, Rapport final de la Commission de vérité et réconciliation du Canada (Montréal/Kingston, McGill/ Queen's University Press, 2015).

4. Ce décompte exclut les écoles résidentielles inuit du Nunavik.

5. Marie-Pierre Bousquet, «Êtres libres ou sauvages à civiliser?», Revue d'histoire de l'enfance "irrégulière», 14 (2012): 62-192; Commission de vérité et réconciliation du Canada, Honorer la vérité, réconcilier pour l'avenir, Sommaire du rapport final de la Commission de vérité et réconciliation du Canada (Winnipeg, Commission de vérité et réconciliation du Canada, 2015); Emanuelle Dufour, «De l’intégration à l'autonomisation; Les fondements idéologiques du père o.m.i. André Renaud, directeur des recherches pour les pensionnats indiens (1954-1963)», dans Marie-Pierre Bousquet et Karl Hele, dir., Spiritual Burn Victims; Native Residential Schools in Quebec/Les grands brûlés de l'âme; les pensionnats autochtones au Québec (Montréal/Kingston, McGill/Queens University Press, à paraître).

6. Sébastien Grammond, Identity Captured by Law. Membership in Canada's Indigenous Peoples and Linguistic Minorities (Montreal/Kingston, McGill/Queen's University Press, 2009), 100-101.

7. Acte pour amender et refondre les lois concernant les sauvages, L.C. 1876, c.18.

8. Acte pour amender et refondre les lois concernant les Sauvages, L.C. 1880, c. 28.

9. Gilles Ottawa, Les pensionnats indiens au Québec, un double regard (Québec, Cornac, 2010).

10. Commission de vérité et réconciliation du Canada, op. cit. 
Depuis quelques années sont documentées les conséquences désastreuses du régime des pensionnats autochtones du Canada pour les générations directement concernées qui héritèrent de nombreux traumatismes physiques, socioculturels, affectifs, identitaires et psychologiques, mais aussi pour les générations précédentes et subséquentes ${ }^{11}$. La déchirure identitaire et culturelle trouva également écho en dehors des murs des pensionnats et eut l'effet de pervertir le rapport à l'éducation institutionnelle de plusieurs communautés autochtones ${ }^{12}$. Ce n'est qu'en 2008 que le gouvernement canadien formula des excuses officielles à l'endroit des peuples autochtones, reconnaissant que la stratégie d'intégration par assimilation à la culture dominante était erronée et néfaste ${ }^{13}$. Quelques années plus tard, en 2015 , la Commission de vérité et réconciliation déposa son rapport final, à l'issue de six années de travaux et d'audiences publiques à travers le pays. En plus de conclure que le régime des pensionnats s'était inscrit dans une tentative de génocide culturel orchestrée par le gouvernement canadien, elle présenta 94 recommandations, ou appels à l'action, adressés à différentes instances gouvernementales, institutionnelles, cléricales et sociales en vue d'un processus de réconciliation des peuples en présence ${ }^{14}$.

Entre-temps, soit entre la fin des années 1960 et le début des années 1970, les États-Unis et le Canada assistèrent à l'essor de mouvements autochtones et de revendications autonomistes notamment associés à la prise en charge de l'éducation. À la suite de la création du Navajo Community College en 1968 émergea le mouvement des Tribal Colleges and Universities (TCU) ${ }^{15}$ dans le but de favoriser l'accès et la réussite des étudiants autochtones à l'intérieur d'un contexte de formation culturellement sécurisant. Les TCU sont, depuis lors, administrés par des conseils majoritairement autochtones

11. Marie-Pierre Bousquet, "A Generation in Politics; The Alumni of the Saint-Marc-de-FigueryResidential School", dans H. C. Wolfart, dir., Papers of the Thirty-Seventh Algonquian Conference (Winnipeg, University of Manitoba, 2006), 3-7.

12. Anne-Laure Bourdaleix-Manin et Marguerite Loiselle, «Le transfert de la souffrance liée à l'institution scolaire; le cas d'une communauté algonquine au Québec», Les Collectifs du Cirp, 2 (2011): 269-286.

13. Stephen Harper, Excuses au nom des Canadiens relativement aux pensionnats, www.pm.gc.ca/ $\mathrm{fra} /$ nouvelles / 2008/06/11/premier-ministre-harper-presente-des-excuses-completes-au-nom-des-canadiens [consulté en mai 2015].

14. Commission de vérité et réconciliation du Canada, Appels à l'action (Winnipeg, Commission de vérité et réconciliation du Canada, 2012).

15. Wayne J. Stein, "Tribal Colleges: A Success Story», New Directions for Community Colleges, 80 (1992): 89-96. Donna Brown, "Tribal Colleges: Playing a Key Role in the Transition from Secondary to Postsecondary Education for American Indian Students", Journal of American Indian Education, 42, 1 (2003): 36-45. 
et situés à même les communautés. Ils visent à offrir une formation postsecondaire dans une certaine adaptation curriculaire, alliant histoires, cultures et traditions autochtones, tout en répondant aux exigences de la formation postsecondaire. En 2016, The American Indian Higher Education Consortium dénombrait plus d'une trentaine de TCU affiliés, répartis sur 75 campus du territoire américain en plus des institutions non affiliées ${ }^{16}$. Du côté des provinces canadiennes telles que l'Ontario, le Manitoba, la Saskatchewan, l'Alberta et la Colombie-Britannique, on note la création de plusieurs dizaines d'établissements postsecondaires inspirés du modèle des TCU. Bon nombre d'entre eux sont regroupés régionalement dans différentes associations comme le Aboriginal Institutional Consortium, le First Nations Adult and Higher Education Consortium ainsi que le Indigenous Adult and Higher Learning Association.

Qu'en est-il du Québec? La mobilisation autochtone a-t-elle également permis d'y développer des structures éducationnelles adaptées aux besoins spécifiques des étudiants autochtones des niveaux postsecondaires? Et si oui, dans quel cadre historique peut-on positionner l'émergence de ces initiatives? L'expérience de Manitou (ou Manitou Community College) de La Macaza (1973-1976) s'est révélée déterminante dans le mouvement d'autonomisation de l'éducation autochtone sur le plan national. Malheureusement très peu d'articles ou d'ouvrages (et encore moins en langue française) ont été réalisés sur le sujet.

À travers le recensement d'informations issues de sources archivistiques et l'apport de nouveaux témoignages d'acteurs clés, cet article propose de retracer les grandes lignes de l'histoire du défunt Collège Manitou. Il vise ainsi à mettre en lumière les liens entre différentes initiatives pionnières ayant mené à sa création ainsi qu'à identifier le caractère innovant et les défis rencontrés par le projet dans le but de contribuer à l'écriture d'un pan

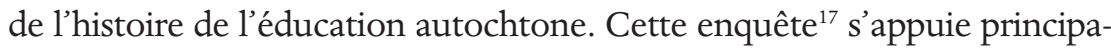
lement sur une méthodologie alliant ethnohistoire et histoire orale, c'està-dire sur des données issues d'entrevues semi-dirigées à durées variables,

16. American Indian Higher Education Consortium, About AIHEC; TRIBAL Colleges; Educating, Engaging, Innovating, Sustaning, www.aihec.org/who-we-are/index.htm [consulté en décembre 2016].

17. Les entrevues semi-dirigées ainsi que la recherche préliminaire ont été réalisées dans le cadre d'un mémoire de maitrise réalisé au département d'anthropologie de l'Université de Montréal sous la direction de Marie-Pierre Bousquet et de Bob W. White. Le chapitre portant sur l'histoire de Manitou a notamment permis de contextualiser l'histoire de la création de l'Institution Kiuna, un institut collégial par et pour les Premières Nations ayant ouvert ses portes dans la communauté d'Odanak en 2011. Emanuelle Dufour, "La sécurité culturelle en tant que moteur de réussite postsecondaire: Enquête auprès d'étudiants autochtones de l'Institution Kiuna et des espaces adaptés au sein des établissements allochtones", mémoire de maîtrise (anthropologie), Université de Montréal, 2015. 
réalisées entre 2014 et 2015, ainsi que sur des témoignages prélevés à même le documentaire "L'éveil du pouvoir ${ }^{18}$ » et le reportage "Vu et entendu sur le Campus au Collège Manitou ${ }^{19}$ ». Le corpus regroupe ainsi les voix de l'un des concepteurs du programme Amérindisation (Rémi Cadieux), des deuxième et troisième directeurs du collège (George Miller et Jacques Kurtness) ainsi que celles d'anciens étudiants, principalement francophones, de l'Institution Manitou (Lise Bastien, Ghislain Picard et Bernard Hervieux).

Cette première cueillette de données a été combinée au débroussaillage de rapports, de documents d'archives, ouvrages et témoignages produits par des individus ayant eux aussi travaillé ou étudié au campus de La Macaza. Parmi ceux-ci, citons notamment l'apport inestimable de l'auteur et professeur Blair Stonechild ${ }^{20}$, membre du McGill Intertribal Council, devenu directeur adjoint du Collège Manitou, mais également ceux de Connie Kilfoil ${ }^{21}$, qui y a conduit une recherche en 1976, de Velma Bourque, qui a occupé les postes de directrice académique et d'employée au MAINC $^{22}$, des enseignants Jean Beaudoin ${ }^{23}$, Domingo Cisneros ${ }^{24}$ et Ernie Benedict $^{25}$ ainsi que des étudiants du collectif Students of Manitou ${ }^{26}$. Une attention particulière a donc été portée à la manière dont ces informateurs donnent un sens aux réalités rapportées et l'énoncent dans un processus de subjectivation essentiel à la construction de l'histoire. Le travail d'analyse s’inspire également de la méthode interactionniste ${ }^{27}$ puisqu'il s'appuie sur l'interprétation propre, ou sur l'univers de référents et de significations des principaux intéressés. L’apport de contributeurs extérieurs a également été intégré pour compléter et supporter le récit.

18. René Sioui Labelle, L'éveil du pouvoir, Bernard Hervieux, Jean-Yves De Banville et Jean Huppé, prod., (Wendake, Production Tshinanu Inc., 2009).

19. Michèle Vincelette et Lyse Carter, «Vu et entendu au Collège Manitou », Recherches amérindiennes au Québec, 5, 4-5 (1975): 85-97.

20. Blair Stonechild, The New Buffalo: the Stuggle for Aboriginal Post-Secondary Education in Canada (Winnipeg, University of Manitoba Press, 2006).

21. Conni Kilfoil, Education and Identity Change:The Manitou Case, mémoire de maîtrise (anthropologie) Université McGill, 1979.

22. Velma Bourque, John Dione et George W. Miller, Special Report on Manitou Community College (Ottawa, Ministèere des Affaires indiennes et du Nord Canada, 1976); Velma Bourque, Projet Amérindisation - Rapport 1972-1979 (Ottawa, Ministère des Affaires indiennes et du Nord Canada, 1979)

23. Jean Beaudoin, «Pourquoi la Macaza», Recherches amérindiennes au Québec, 6, 3-4 (1976): 29-32.

24. Diego Cisneros, «Les lieux sauvages», Urgences, 17-18 (octobre 1987): 19-27.

25. E. Benedict, cité dans M. Vincelette et L. Carter, loc. cit., 90-91.

26. Students of Manitou, Hear Our Words (La Macaza, ThunderBird Press, 1976).

27. Joëlle Morrissette, Sylvie Guingnon et Didier Demazièere, «De l’usage des perspectives interactionnistes en recherche», Recherches qualitatives, 30, 1 (mai 2011): 1-9 


\section{EN ROUTE VERS LA MAÎTRISE INDIENNE DE L'ÉDUCATION INDIENNE}

Dans les années 1960, le Canada comptait environ deux cents étudiants issus de Premières Nations inscrits au sein d'institutions d'éducation supérieure ${ }^{28}$. Le Québec se classait en tête de liste des provinces canadiennes avec trentecinq étudiants d'origine autochtone au sein de ses universités en $1963^{29}$.

Considérant que les tentatives en matière d'éducation autochtone s'étaient jusqu'alors révélées infructueuses, le père André Renaud, o.m.i., instaura, pour le compte de la University of Saskatchewan, le Indian and Northern Education Program (INEP) en 1961. Renaud proposait une toute nouvelle approche de l'éducation autochtone inspirée par un renversement de paradigme. Impliqué de près dans l'implantation du régime des pensionnats autochtones au Québec en tant que directeur des études à la Commission oblate des affaires indiennes et esquimaudes, Renaud posait désormais un regard critique sur cette expérience. Il défendit alors l’idée que la persévérance et la réussite scolaires des étudiants autochtones devaient dorénavant passer par la promotion de l'identité culturelle et une formation adéquate des maîtres appelés à travailler en contextes autochtones $^{30}$. L'INEP, en tant que projet expérimental, devint ainsi l'un des premiers programmes d'éducation postsecondaire adapté aux réalités culturelles autochtones et eut une incidence marquée sur le développement d'initiatives connexes dans l'est du pays.

Le rapport Hawthorn-Tremblay fut publié quelques années plus tard, soit en 1967. Cette commission s'inscrivait en réponse à une demande d'enquête du ministère de la Citoyenneté et de l'Immigration sur les Indiens du Canada. Dans son deuxième volume "Besoins et mesures d'ordre économique, politique et éducatif ", la scolarisation des élèves autochtones était décrite comme une expérience bouleversante sur le plan identitaire dans ses visées assimilatrices ${ }^{31}$. Pour tenter de remédier à ce problème, les auteurs formulèrent soixante recommandations, insistant notamment sur l'importance de valoriser les cultures autochtones au sein de l'éducation et d'encourager l'implication parentale autochtone dans la gestion des institutions scolaires ${ }^{32}$. Le rapport permit également l'émer-

28. David Holmes, Embrassing Differences; Post-Secondary Education among Aboriginal Students, Students with Children and Students with Disabilities (Ottawa, The Canada Millennium Foundation, 2005).

29. Anonyme, «Indiens du Québec à l'Université», Vie indienne, 2, 20 (1963): 7.

30. André Renaud, "An Experiment in Curriculum Change», OISE Conference on Canadian Studies (Toronto, 22 février 1969); E. Dufour, «De l'intégration à l'autonomisation... », loc. cit.

31. Harry B. Hawthorn et Marc-Adélard Tremblay, Étude sur les Indiens contemporains du Canada; Besoins et mesures d'ordre économique, politique et éducatif - Deuxième partie (Ottawa, Indian affairs, 1967).

32. Ibid. 
gence de la notion de "Citizens Plus », qui rétablissait le concept d'égalité en critiquant l'égalitarisme d'après-guerre et reconnaissait des droits spécifiques aux citoyens autochtones. En 1970, ce concept fut récupéré par le réquisitoire de l'Indian Association of Alberta, intitulé Citizens Plus (ou le Livre rouge) en référence au rejet en bloc des propositions du Livre blanc du gouvernement Trudeau ${ }^{33}$. Le Livre rouge plaidait notamment pour une révision de la Loi sur les Indiens ainsi qu'une "adéquation entre les programmes scolaires et les valeurs autochtones ${ }^{34}$.

C'est donc dans un climat politique foisonnant qui eut pour effet de mobiliser divers groupes autochtones que fut publiée, en 1972, La Maîtrise indienne de l'éducation indienne par la Fraternité des Indiens du Canada $(\mathrm{FIC})^{35}$. Le document en appelait principalement à la réappropriation administrative et culturelle de l'éducation par les Autochtones en définissait les grandes lignes de «la doctrine, les objectifs, les principes et l'orientation qui dev[aient] régir tous les programmes scolaires indiens ${ }^{36} »$ :

Il est temps d'apporter un changement radical à l'éducation dispensée aux Indiens. Nous visons un système d'éducation qui correspond à la philosophie et aux besoins des Indiens. Nous voulons que l'éducation contribue au sens de l'identification et de la confiance en soi chez nos enfants ${ }^{37}$.

L'accord de principe présentait un profond enracinement dans les valeurs culturelles et la fierté identitaire. La Fraternité des Indiens du Canada proposait ainsi des mesures adaptatives particulièrement associées à quatre domaines essentiels : la responsabilité juridique et administrative, les programmes pédagogiques, l'enseignement et ses langues ainsi que les installations et services. Elle préconisait notamment la formation d'enseignants autochtones, une meilleure représentativité des langues autochtones dans l'enseignement ainsi que des services et installations adaptés aux besoins spécifiques de la population locale ${ }^{38}$. En matière

33. Publié en 1969 par le ministre des Affaires indiennes et du Nord Canada, Jean Chrétien, le Livre blanc sur la politique indienne visait à abolir le titre indien, la Loi sur les Indiens et les droits acquis dans une perspective soi-disant égalitariste pour faire des Autochtones des citoyens comme les autres Canadiens (Canada, La politique indienne du Gouvernement du Canada [Livre blanc sur la politique indienne] (Ottawa, Ministère des Affaires indiennes et du Nord Canada, 1969).

34. Aurélie Hot, «L'école des Premières nations au Québec », Cahier DIALOG, 10 (Montréal, INRS et DIALOG, 2010): 17.

35. La Fraternité des Indiens du Canada est l'instigatrice de l'Assemblée des Premières Nations (APN) qui sera créée en 1982.

36. La Fraternité des Indiens du Canada, La Mâ̂trise indienne de l'éducation indienne (Ottawa, Fraternité des Indiens du Canada, 1972), iii.

37. Ibid., 3.

38. Ibid. 
d'éducation postsecondaire, La Maîtrise indienne de l'éducation indienne souhaitait favoriser la diplomation d'étudiants autochtones dans le but de combler les besoins professionnels des communautés (avocats, médecins et enseignants). Le document revendiquait aussi un soutien financier adéquat, un plan de recrutement ciblé et des politiques d'admission adaptées aux réalités des Premières Nations:

Vu le grand besoin de professionnels dans les agglomérations indiennes, il faut encourager et aider par tous les moyens possibles les Indiens désireux de poursuivre des études supérieures [...]. Compte tenu de tous les désavantages subis par les Indiens dans le domaine de l'éducation, les conditions rigides actuelles d'admission aux universités et aux collèges devraient être adaptées ${ }^{39} \ldots$

La Maîtrise indienne de l'éducation indienne s'articulait comme une volonté de promotion identitaire et d'autonomisation facilitée par la réappropriation des instances éducatives. Sa philosophie s'inscrivait en ligne directe avec le renversement de paradigmes proposé par l'Indian and Northern Education Program, le Livre rouge et les perspectives exposées par le rapport HawthornTremblay $^{40}$. Selon Velma Bourque, l'adoption de cet accord de principe par le ministre des Affaires indiennes de l'époque, Jean Chrétien, eut l'effet d'officialiser un processus entamé au Québec depuis la nomination, en 1970, d'Aurélien Gill au poste de premier directeur autochtone de l'éducation régionale de l'histoire du ministère des Affaires indiennes et du Nord Canada (MAINC) $)^{41}$. En outre, cette reconnaissance gouvernementale fut à l'origine du financement subséquent de centres d'éducation culturelle créés sur le modèle proposé par Citizen Plus et la Maîtrise indienne de l'éducation indienne ${ }^{42}$ :

C'est par des études sur l'histoire, la culture, la langue et les valeurs indiennes que les Centres d'éducation culturelle seront utiles en ce sens. En apprenant comment utiliser ses croyances, ses valeurs et ses talents traditionnels pour survivre dans la société moderne et en apprenant les techniques et les comportements modernes nécessaires pour participer à la vie économique et sociale, l'Indien gagnera en assurance et en indépendance. Les Centres d'éducation culturelles $[s i c]$ seront conçus de façon à répondre à ces besoins et à remédier aux faiblesses des autres programmes d'enseignement ${ }^{43}$.

\footnotetext{
39. Ibid.,14.

40. A. Hot, «L'école des Premières nations au Québec», loc. cit.; E. Dufour, «De l'intégration à l'autonomisation...", loc. cit.

41. V. Bourque, Projet Amérindisation..., op. cit.

42. B. Stonechild, The New Buffalo..., op. cit.

43. FIC, La maîtrise indienne ..., op. cit., 17.
} 


\section{CRÉATION DU PROGRAMME AMÉRINDISATION}

En 1971, le McGill Intertribal Council of Native Students ${ }^{44}$ mettait sur pied le Native North American Studies Institute (NNASI), ou l'Institut d'études des aborigènes d'Amérique du Nord, en collaboration avec l'association des Indiens du Québec $(\mathrm{AIQ})^{45}$. Le NNASI visait à offrir aux jeunes Autochtones une éducation culturelle capable de favoriser l'émergence d'une fierté identitaire à l'intérieur des communautés, tout comme en contexte urbain ${ }^{46}$.

...the proposed N.N.A.S.I. will enable native North American students and other native people in Eastern Canada and the Eastern Arctic to study their own languages, their history, and cultures while obtaining knowledge and modern technical skills needed to gain "control of their economic, social, and political destiny" ${ }^{47}$.

William Craig, un citoyen américain invoquant des origines mohawks ${ }^{48}$ et impliqué dans la fondation du Indian Studies Program de la University of Minnesota ${ }^{49}$ fut engagé pour diriger le N.N.A.S.I. L'institut était notamment financé par les subventions ponctuelles de la Fondation McConnell, d’un programme jeunesse du secrétaire d'État, du Collège Dawson et du MAINC $^{50}$.

44. Le McGill Intertribal Council of Native Students a émergé au début des années 1970 à la suite d'une discussion publique organisée sur le campus et portant sur le thème du Livre blanc du gouvernement fédéral. Il est à l'origine de l'organisation d'une rencontre au Loyola College au printemps suivant autour de la question de l'éducation autochtone puis d'un premier cours portant sur les réalités autochtones du Canada enseigné par Gail Valaskakis. Il visait à réunir tous les étudiants autochtones des villes de Montréal et de Québec pour répondre à leurs besoins culturels. Son plan d'action fut reconnu et soutenu par les Universités McGill, Loyala et Sir George Williams et quelques cégeps anglophones de Montréal. Malgré la mobilisation de certains de leurs étudiants autochtones et des membres de leur personnel, les universités francophones de Montréal et de Québec s'abstinrent de toute reconnaissance officielle. J. E. Bernard, Report of Review Committee: Manitou College, octobre 1975, Bibliothèque et Archives Canada (BAC), RG10C-IV-9, 1996-97/978,19, 24: C. Kilfoil, Education and Identity..., op. cit..

45. Le conseil d'administration du NNASI était formé par des professeurs, des étudiants ainsi que des représentants de l'Association des Indiens du Québec et du Northern Quebec Inuit Association (NQIA). B. Stonechild, The New Buffalo..., op. cit.; BAC, RG10-C-IV-9, 1996-97/978,19, 23.

46. Anaïs Janin, «Le Collège Manitou (1973-1976), un premier exemple d'éducation postsecondaire en contexte autochtone au Québec", dans C. Levesque et B. Sioui, dir., Parlons de l'éducation des Autochtones au Québec : 12-18, Cahiers DIALOG, 2 (Montréal, DIALOG et INRS, 2011).

47. McGill University Intertribal Council of Native Students, 1970, dans C. Kilfoil, Education and Identity Change..., op. cit., 22.

48. Les origines autochtones de Craig furent par la suite contestées. Denis Patrick Foley, «L'éducation des minorités", Recherches amérindiennes au Québec, 6, 3-4 (1976): 33-38.

49. BAC, RG10-C-IV-9, 1996-97/978, 24. Ledit programme servit notamment de modèle pour l'élaboration des programmes culturellement adaptés de Manitou alors que Craig, Renaud et Bourque se rendirent à deux reprises à la University of Manitoba pour s'inspirer de ses activités. Ibid, 23.

50. BAC, RG10-C-IV-9, 1996-97/978, 19, 23. 
Dès 1971, le NNASI veilla à offrir des programmes de formation pour les étudiants autochtones et des cours de création de matériel pédagogique autochtone pour les écoles dans les locaux du Collège Dawson et de l'Université McGill51 ${ }^{5}$. Une douzaine d'étudiants cris de la Baie James firent ainsi leur entrée à McGill dans le cadre d'un programme spécial de formation des maitres-assistants autochtones ${ }^{52}$. Des cours destinés aux professeurs appelés à enseigner dans les communautés autochtones furent également organisés dans la ville de Québec ${ }^{53}$.

Dans les deux années suivant le début du programme de formation des maîtres de McGill, les étudiants des deux cohortes cries furent redirigés vers le niveau collégial pour l'obtention de formations préalables ${ }^{54}$. Alors qu'à la demande du directeur régional innu, Aurélien Gill ${ }^{55}$, le MAINC rédigeait une version préliminaire du projet d'Amérindisation des écoles ${ }^{56}$, André Renaud déposa, pour le compte de l'Université du Québec à Chicoutimi (UQAC), un rapport inspiré par les idées novatrices du Indian and Northern Education Institute à l'intention de la sous-ministre de l'Éducation du Québec $^{57}$. En juillet 1972, l'UQAC se vit confier le programme de formation des maîtres autochtones de langue française et anglaise, engageant ainsi le transfert du programme entamé par l'Université McGills8. Ce mandat fut mis en œuvre par l'organisation d'une première école d'été à Chicoutimi ${ }^{59}$. Parallèlement, la Federation of Saskatchewan Indian Nations, aidée par André Renaud, inaugurait la même année le Saskatchewan Indian Cultural College à l'University of Saskatchewan, subventionné par un nouveau fonds d'éducation culturelle mis en place par le ministre des Affaires indiennes et du Développement du Nord, Jean Chrétien, et le secrétaire d'État, Gérard Pelletier $^{60}$. Le 26 octobre 1972, le NNASI se vit attribuer une première subvention dans le cadre de la phase initiale du programme ${ }^{61}$.

51. V. Bourque, Projet Amérindisation..., op. cit.; A. Janin, «Le Collège Manitou... », loc. cit.

52. V. Bourque, Projet Amérindisation.., op. cit.; BAC, RG10-C-IV-9, 1996-97-978,14; J. E. Bernard, Report of Review..., op.cit.

53. J. E. Bernard, A review of..., op.cit.

54. V. Bourque et al., Special Report on Manitou Community College..., op. cit.

55. Le plan quinquennal est déposé en 1973. V. Bourque, Projet Amérindisation..., op. cit.

56. Ce plan s'articule principalement autour de l'intégration d'enseignants, de langues et de contenus autochtones à même le système d'éducation. Projet d'Amérindinsation des écoles, Premier plan quinquennal, brouillon, 1972. BAC, RG10-C-IV-9, 1996-97/978, 10.

57. André Renaud, Projet en éducation des Indiens et Esquimaux du Québec (Chicoutimi, Université du Québec à Chicoutimi, 1972); BAC, RG10-C-IV-9, 1996-97/978, 14.

58. V. Bourque, Projet Amérindisation..., op. cit.; BAC, RG10-C-IV-9, 1996-97/978, 25.

59. Ibid.

60. B. Stonechild, The New Buffalo..., op. cit., 2006; SICC History, www.sicc.sk.ca/history_css.html [consulté en janvier 2017]; BAC, RG10-C-IV-9, 1996-97/978, 25.

61. BAC, RG10-C-IV-9, 1996-97/978, 23. 
Entre 1972 et 1973, l'AIQ et le NNASI - lequel projetait de se délocaliser des grands centres urbains pour s'agrandir et aplanir certains obstacles adaptatifs $^{62}$ - obtinrent officiellement les droits d'occupation de la base militaire désaffectée de la Bomarc Missile, à La Macaza, par le concours du ministère des Affaires indiennes ${ }^{63}$. Bien que les installations eussent été laissées à l'abandon depuis plusieurs années au moment de la transaction, Kilfoil rapporte que celles-ci étaient néanmoins estimées à près de 60 millions de dollars ${ }^{64}$. Le NNASI, à la suite d'une étude de faisabilité ${ }^{65}$ déposée en août 1972, avait préalablement conclu que le site était idéal pour la création d'un centre d'éducation culturelle inspiré du modèle du Navajo Community College d'Arizona ${ }^{66}$. Ces démarches furent appuyées par Renaud qui avait lui-même participé, près de dix années auparavant $t^{67}$, à un stage d'observation au College of Education de l'Université de l'Arizona à Phoenix pour "y étudier les méthodes d'enseignement, de pédagogie et de recherche en anthropologie appliquée à l'éducation des Indiens du Sud-Ouest américain ${ }^{68} \%$.

\section{LA GENÈSE DU PROJET MANITOU}

Entre 1972 et 1973, la base militaire de la Macaza devint ainsi le Collège Manitou (ou le Manitou Community College), première institution d'enseignement postsecondaire du Québec conçue spécialement pour la formation d'étudiants autochtones venus des quatre coins du Canada. À la tête du NNASI, William Craig hérita de la direction de l'établissement administré par un conseil des gouverneurs (Board of Governors) ${ }^{69}$.

62. J. E. Bernard, Review of..., op.cit.; BAC, RG10-C-IV-9, 1996-97/978, 23.

63. V. Bourque, Projet Amérindisation..., op.cit., 1979; B. Stonechild, The New Buffalo..., op. cit. Le MAINC fit l'acquisition de la base pour 539000 \$ au mois de décembre 1972. BAC, RG10-C-IV-9, 199697/978, 23.

64. C. Kilfoil, Education and Identity Change..., op. cit.

65. BAC, RG10-C-IV-9, 1996-97/978, 23.

66. B. Stonechild, The New Buffalo..., op. cit. Tel qu'évoqué un peu plus tôt, le Navajo Community College constitue un pionnier de la mouvance des Tribal Colleges and Universities américains. Il est aujourd'hui devenu le Diné College. Il restera un modèle référentiel pour le collège Manitou et une correspondance étroite sera entretenue avec celui-ci sous la direction de George Miller; BAC, RG10C-IV-9, 1996-97/978, 14, 22, 34.

67. Soit huit années après le passage de l'anthropologue Marc-Adélard Tremblay, coauteur du Rapport Hawthorn-Tremblay, dans la même aire culturelle.

68. Anonyme, "Attends Arizona University», Indian Record, 26, 2 (mars-avril 1963): 8, traduction libre.

69. C. Kilfoil, Education and Identity Change..., op. cit. À partir de 1975, le conseil d'administration était composé de représentants de chacune des nations autochtones du Québec finançant le projet. B. Stonechild, The New Buffalo..., op. cit.; BAC, RG10-C-IV-9, 1996-97/978, 30. 
L'aventure de Manitou fut officialisée par le déménagement du programme de formation des maîtres autochtones de l'UQAC à La Macaza ${ }^{70}$. Le père oblat Rémi Cadieux, qui avait notamment dirigé le pensionnat d'Amos entre 1968 et 1972 et possédait une grande expérience en milieux autochtones, fut mandaté par l'UQAC pour l'élaboration d'un programme officiel de formation des maîtres pour les Premières Nations du Québec ${ }^{71}$. Inspiré par les observations et expériences recueillies en tant qu'enseignant-observateur à l'Indian and Northern Education Program ${ }^{72}$, Cadieux travailla en collaboration avec la directrice du module en éducation de l'UQAC, sœur Jeannine Lapierre de la congrégation Notre-Dame-du-BonConseil, à l'élaboration du cursus ${ }^{73}$. Le programme devait offrir une formation adaptée aux réalités et cultures des Premières Nations et fournir des outils multidisciplinaires à ses étudiants. Il était ainsi question de permettre «une éducation plus humaine et plus efficace grâce à une adaptation adéquate aux langues et aux cultures et faire de l'école un gardien de la survie ethnique ${ }^{74} \gg$. L'objectif principal était donc de former des aidesenseignants et des professeurs de langues autochtones dans le but de contribuer au projet d'Amérindisation des écoles ${ }^{75}$. Le programme reçut l'approbation du ministère de l'Éducation du Québec (MEQ), du Sénat du Canada, du ministère des Affaires indiennes du Canada et du Nord Canada (MAINC) et de ' $^{\prime} \mathrm{AIQ}^{76}$.

Le $1^{\text {er }}$ juillet 1973, le campus du Collège Manitou recevait sa première cohorte composée de quatre-vingts étudiants pour une première école d'été de six semaines ${ }^{77}$. Elle eut pour effet transversal de faire connaitre la nouvelle institution à l'intérieur des communautés - parallèlement au programme de recrutement du Collège Manitou initié par le NNASI et financé par le $\mathrm{MAINC}^{78}$ - et de préparer le terrain pour l'inauguration du nouveau programme de sciences sociales accrédité par le Collège Dawson en septembre

70. V. Bourque, Projet Amérindisation..., op. cit., 7.

71. Entrevue avec Rémi Cadieux, le 16 décembre 2014; V. Bourque, Projet Amérindisation..., op. cit.

72. Entrevue avec Rémi Cadieux, le 5 janvier 2015.

73. Ibid.

74. Parmi les cours offerts au programme Amérindisation figuraient notamment les intitulés suivants: «Enseignement en langues amérindiennes, l’Amérindien et son environnement naturel, Expression et communication, Innovations pédagogiques et l'Amérindien, Préparation et organisation du matériel didactique, Enseignement amérindien et autres systèmes d'éducation, Processus d'apprentissage amérindien, Linguistique amérindienne ainsi que Pratique d'enseignement ». C. Kilfoil, Education and Identity Change..., op. cit., 25, traduction libre.

75. V. Bourque, Projet Amérindisation..., op. cit.

76. Entrevue avec Rémi Cadieux le 16 décembre 2014.

77. V. Bourque, Projet Amérindisation..., op. cit.; J. E. Bernard, Report of..., op. cit.

78. BAC, RG-10-C-IV-9, 1996-97-978, 23. 
$1973^{79}$. Le Collège Manitou était principalement financé par le Programme de fonds d'éducation culturelle, une allocation per capita octroyée aux bandes par le MAINC ${ }^{80}$. C'est donc sur une base volontaire que les nations avaient décidé d'investir dans le financement du collège autochtone ${ }^{81}$. L’histoire du Collège Manitou révéla que la structure budgétaire des centres d'éducation culturelle, qui reposait entièrement sur l'investissement discrétionnaire de chacun des partis, eut l'effet de fragiliser notablement ledit projet. De plus, ces fonds se révélèrent insuffisants pour couvrir le coûteux entretien des installations et infrastructures de la base militaire, la production de matériel didactique culturellement adapté, le personnel enseignant et de soutien ainsi que les autres activités du collège ${ }^{82}$. Malgré l'ajout d'un financement provincial dès 1974 et de certaines bourses fédérales non récurrentes ${ }^{83}$, l'administration se vit donc dans l'obligation de voir à la production de "revenus autogénérés ${ }^{84}$ » à travers la sollicitation de fondations et même, ultimement, d'entreprises privées ${ }^{85}$.

Dès les mois qui suivirent son ouverture, le Collège Manitou fit face à une situation financière critique ${ }^{86}$. Le budget déficitaire occasionna des dissensions internes autour de la gestion du collège ${ }^{87}$ et William Craig quitta son poste de directeur fondateur en novembre $1973^{88}$. Alors que Stonechild évoque des raisons médicales afin d'expliquer le soudain changement de direction ${ }^{89}$, le père Cadieux raconte que l'AIQ l'aurait chargé d'aller obtenir sa démission ${ }^{90}$. Cette dernière thèse est corroborée par une entrevue accordée par Craig à un journal local dans lequel il attribua sa démission au fait qu' «il ne pouvait accepter qu'on fasse du collège

79. Le programme d'études collégiales comprenait les cours de tronc commun suivants: anglais, humanités et éducation physique. Des cours à option tels que «administration, anthropologie, sociologie, économie, français, histoire, mathématiques, langues amérindiennes, philosophie et psychologie» figuraient également au cursus. C. Kilfoil, Education and Identity Change..., loc. cit., 25-26, traduction libre.

80. B. Stonechild, The New Buffalo..., op. cit.; Entrevue avec Jacques Kurtness, le 24 novembre 2015.

81. J. E. Bernard, A review of..., op.cit.

82. V. Bourque et al., Special Report on Manitou Community College..., op. cit., 3 ; J. Beaudoin, «Pourquoi la Macaza ", loc. cit., 30-31; B. Stonechild, The New Buffalo..., op. cit.; Entrevue avec Jacques Kurtness, le 24 novembre 2015.

83. Claude Harmegnies, «Les bandes indiennes laissent tomber le collège Manitou », Le Devoir, 20 décembre 1976; B. Stonechild, The New Buffalo..., op. cit.; J. E. Bernard, Report of..., op. cit.

84. V. Bourque et al., Special Report on Manitou Community College..., op. cit., 6

85. BAC, RG10-C-IV-9, 1996-97-978, 11-12-13-18-24.

86. BAC, RG10-C-IV-9, 1996-97-978, 24, 19.

87. Entrevue avec Rémi Cadieux, le 5 janvier 2015 ; J. E. Bernard, Report of..., op. cit.

88. Ibid.

89. B. Stonechild, The New Buffalo..., op. cit., 56

90. Entrevue téléphonique avec Rémi Cadieux, le 16 décembre 2014. 
Manitou, un autre collège blanc $»^{91}$. Quelles qu'en soient les raisons, ce départ souleva d'importants remous chez les étudiants et le personnel autochtone du collège qui lui étaient attachés ${ }^{22}$. Environ 175 étudiants autochtones fréquentaient alors Manitou ${ }^{93}$. George W. Miller, anthropologue et membre de la nation mohawk de Six Nations of the Grand River en Ontario, hérita de la direction générale du collège. Le second directeur de Manitou «fit face à deux principaux défis; établir la stabilité financière et la crédibilité académique du collège $»^{94}$.

L'année qui suivit enregistra un bilan budgétaire exceptionnellement positif. Les efforts du nouveau directeur conjugués à l'embauche d'un contrôleur-comptable et à la diminution des effectifs permirent d'éponger en bonne partie la dette accumulée en un peu plus d'un $a^{95}$. Les taux de rétention des étudiants, évalués à $74 \%{ }^{96}$, contrastaient fortement avec ceux des étudiants autochtones inscrits dans les universités francophones du Québec $^{97}$. L'Union of Nova Scotia Indians attribua ce succès au fait que l'éducation prodiguée à Manitou amenait les étudiants à renforcer leur identité culturelle et à construire une meilleure confiance en eux-mêmes ${ }^{98}$. On peut postuler que les installations du campus, dont il sera question un peu plus tard, offraient également l'avantage d'aplanir les obstacles inhérents à l'éloignement géographique, favorisant ainsi la persévérance scolaire des étudiants inscrits dans un modèle apparenté à celui des TCU. Le programme collégial comptait alors 122 étudiants, principalement originaires du Québec, des Maritimes et de l'Ontario ${ }^{99}$. Selon Stonechild, la baisse de fréquentation enregistrée entre 1973 et 1974 était principalement associée à l'insuccès académique de certains étudiants ${ }^{100}$.

Le programme collégial francophone fut créé en septembre 1974 sur le canevas de son homologue anglophone. Le cursus accrédité par le Cégep Ahuntsic fut dès lors formellement reconnu par le MEQ. Le gouvernement provincial mandata également le Collège Manitou pour le

91. Anonyme, «Le Dr. Craig s'explique», L'écho de la Lièvre, mercredi 12 décembre 1973, 3.

92. Entrevue avec Rémi Cadieux, le 5 janvier 2015 ; BAC, RG10-C-IV-9, 1996-97-978, 18

93. B. Stonechild, The New Buffalo..., op. cit.

94. Ibid., 56, traduction libre.

95. J. E. Bernard, Report of..., op. cit.

96. Ibid., 57; C. Kilfoil, Education and Identity Change..., op. cit.

97. En 1972, le NNASI estimait ce taux à 10\%. B. Stonechild, The New Buffalo..., op. cit.

98. B. Stonechild, The New Buffalo..., op. cit., 56.

99. Ibid.

100. Ibid. 
développement de cours à contenus autochtones qui seraient ultérieurement dispensés dans les autres cégeps de la province ${ }^{101}$.

Dans les mois qui suivirent, l'artiste mexicain Domingo Cisneros mit sur pied des cours de communication, de théâtre et d'arts autochtones ${ }^{102}$. Ces nouveaux cours à option furent ajoutés au programme de sciences humaines, en plus des cours de biologie, musique, photographie, poterie et religion. Le nouveau programme anglophone de Native Arts and Communication ouvrant sur une diplomation collégiale préuniversitaire ou technique émise par le Collège Dawson fut lancé en août 1975, suivi par la création d'un programme homologue francophone, accrédité par le Cégep Ahuntsic ${ }^{103}$. Des discussions furent également amorcées pour la création d'un département de langues autochtones ${ }^{104}$.

Au cours de cette période, Lise Bastien, future directrice du Conseil en éducation des Premières Nations (CEPN), partit en reconnaissance à La Macaza accompagnée de quelques amis ${ }^{105}$. Le petit groupe de Wendake fit une partie du trajet en autobus, puis compléta le voyage "sur le pouce», attiré par l'occasion d'aller à la rencontre de nouveaux groupes de jeunes Autochtones originaires de différentes nations et communautés ${ }^{106}$. Séduite par l'ambiance communautaire, comme par la perspective d'y recevoir une éducation adaptée à sa réalité, la jeune Huronne-Wendate décida de retourner sur les bancs d'école pour intégrer la cohorte francophone du programme de sciences sociales du Collège Manitou.

\section{UN VILLAGE INDIEN À LA MACAZA, OU L'ÉMERGENCE D'UNE NOUVELLE IDENTIFICATION COLLECTIVE}

La base du Bomarc Missile de La Macaza s'étendait sur une superficie de plus de $1,85 \mathrm{~km}^{2}$. Le Collège Manitou avait hérité de 173 édifices et autres installations laissés à l'abandon, comprenant des salles de cours, des bureaux administratifs, des centres communautaires, une école primaire, une garderie, une bibliothèque, une imprimerie, des studios d'enregistrement, une chambre noire, un centre sportif avec piscine, des terrains de tennis, de baseball, de football, une patinoire, une allée de quilles, un ring de boxe, un bureau de poste, deux serres, un restaurant, un cinéma, un

101. C. Kilfoil, Education and Identity Change..., op. cit.; J. E. Bernard, Report of..., op. cit.

102. A. Janin, «Le College Manitou (1973-1976)», op. cit.

103. BAC, RG10-C-IV-9, 1996-97/978, 17.

104. BAC, RG10-C-IV-9, 1996-97/978, 18.

105. Entrevue avec Lise Bastien, 18 août 2014.

106. Ibid. 


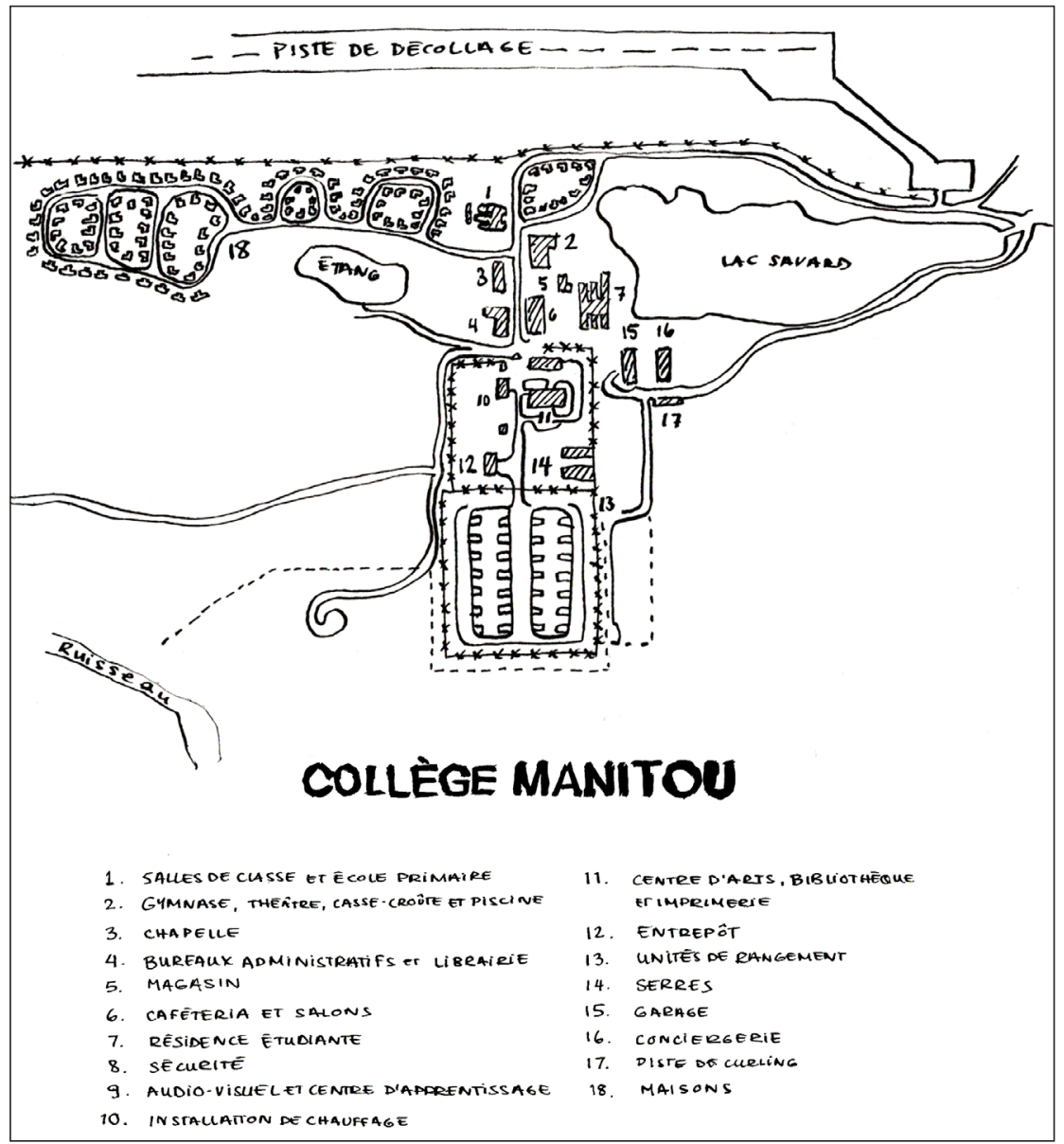

Plan francisé du campus de La Macaza. Dessin et traduction par l'auteure d'après plan contenu dans J. E. Bernard, Report of Review Committee: Manitou College, octobre 1975, p. 198-199. BAC, RG10-C-IV-9, 1996-97/978, 19.

gymnase, un terrain de golf, un magasin, un bar, une chapelle, un cassecroûte, etc. ${ }^{107}$.

Le site permettait en outre d'accueillir les familles et enfants des étudiants et du personnel dans les quelque 114 maisons préfabriquées, d'abord construites pour les familles des militaires:

On vivait comme sur une communauté. On avait recréé l'esprit communautaire; tout le monde ensemble qui se parle, qui se voisine... C'était une base 
militaire et donc, c'était un village en soi. Un village complètement autonome avec toutes les infrastructures qui recréent la communauté [...]. Il y avait aussi les arts, les activités parascolaires, tout ça autour ${ }^{108}$ !

Selon Cadieux, c'était précisément dans cet esprit de réseautage que s'inscrivait l'un des principaux objectifs de Manitou ${ }^{109}$. L'AIQ souhaitait alors permettre à la jeunesse des Premières Nations de se rencontrer. Regroupés en association, les leaders autochtones avaient compris que seul un front commun pouvait leur permettre de faire valoir leurs droits de manière efficace contre la machine gouvernementale. L'identification autochtone représentait, et représente toujours, une force politique et économique contribuant à accroître les pouvoirs de chaque nation ${ }^{110}$. Or, s'il est difficile d'en arriver à un consensus à l'intérieur d'un petit groupe d'individus appartenant à une même communauté (qu'elle soit autochtone ou allochtone), il va sans dire que la collaboration entre les acteurs de différentes nations et communautés nécessitait d'abord qu'ils apprennent à se connaître et à se reconnaître. Alors que les Premières Nations se trouvaient réparties sur un immense territoire sans moyens de communication efficaces, le Collège Manitou représentait une occasion idéale pour créer de nouveaux liens et une identification commune.

S’intéressant au processus d'autonomisation autochtone au Québec, le documentaire L'éveil du pouvoir de René Sioui Labelle (2009) ${ }^{111}$ est riche en archives visuelles et en témoignages permettant de retracer le phénomène Manitou. Son coproducteur Bernard Hervieux, ancien étudiant de Manitou qui est par la suite devenu directeur général de la Société de communication Atikamekw-Montagnais, y explique notamment que les rencontres et les échanges occasionnés par le Collège Manitou ont participé à l'essor d'une nouvelle entité amérindienne: «Je me suis retrouvé au Collège Manitou. Il y avait rien que des Indiens-là [...]. C’est là qu'on nous a amérindianisés [...]. Nous commencions à prendre conscience de qui nous étions ${ }^{112} \ldots$... Il insiste sur le fait que jusqu'alors son parcours scolaire "dans le monde des Blancs» ne lui avait jamais permis un tel essor identitaire. Selon Ernie Benedict, professeur de philosophie de l'éducation, de religion et de cultures indiennes au Collège Manitou, l'objectif de ce type d'enseignement était d'encourager

108. Entrevue avec Lise Bastien, 18 août 2014.

109. Entrevue avec Rémi Cadieux, le 16 décembre 2014.

110. C. Kilfoil, Education and Identity Change..., op. cit.

111. R. Sioui Labelle, «L'éveil du pouvoir», loc. cit.

112. Bernard Hervieux, dans ibid. 
les étudiants à examiner leur patrimoine culturel sous différents angles ${ }^{113}$. Il était alors question d'établir des liens entre les concepts culturels des différentes nations en présence ainsi qu'avec la culture dominante de l'époque. Les interactions communautaires, le collectivisme et le positionnement par rapport à la société allochtone au sein de Manitou contribuèrent à l'émergence d'une nouvelle identité panindienne et rassembleuse ${ }^{114}$. Si cette nouvelle identité n'a pas été intégrée par tous les étudiants de Manitou $^{115}$, chacun fut néanmoins appelé à réfléchir et à se positionner sur les enjeux culturels à travers un processus d'identification collective et d'identisation individuelle ${ }^{116}$. En plus d'être conscientisés aux réalités vécues par différentes nations et communautés et de développer un sentiment de solidarité, les apprenants participaient également à la construction d'une nouvelle culture commune et situationnelle, constituée d'emprunts culturels et d'appropriations. Pour le directeur George Miller, tout comme pour la directrice académique, Velma Bourque ${ }^{117}$, cette avenue panindienne constituait une réponse expérimentale au contrôle de l'éducation "par les autochtones, dans un milieu autochtone»:

La plupart [des jeunes] traversent actuellement une crise d'identité où ils voient leur vie comme un amalgame d'aspects autochtones et une grande proportion d'influences occidentales et cela crée une confusion totale. Elle peut donner naissance à un potentiel pathologique qui se manifeste par le découragement, l'abandon des études, l'abus d'alcool ou de drogues, etc. Ce que nous essayons de faire ici, c'est de leur donner les outils nécessaires pour qu'ils soient en mesure de confirmer leur identité [...]. Idéalement, il faudrait peut-être un système d'éducation différent pour chaque tribu. Nous tenons quand même compte des besoins locaux des populations en offrant certains cours spécialisés à l'intention de groupes précis. Mais le but principal, c'est quand même l'éducation des Indiens de tout le Canada et même des États-Unis ${ }^{118}$.

113. Ernie Benedict, cité dans M. Vincelette et L. Carter, «Vu et entendu au Collège Manitou », loc. cit., 90-91.

114. C. Kilfoil, Education and Identity Change..., op. cit., 46.

115. Ibid., M. Vincelette et L. Carter, "Vu et entendu au Collège Manitou », loc. cit.

116. Louis-Jacques Dorais, "La construction de l'identité», dans Denise Deshaies et Diane Vincent, dir., Discours et construtions identitaires (Québec, Les Presses de l'Université Laval, 2004), 1-11.

117. Velma Bourque, citée dans M. Vincelette et L. Carter, "Vu et entendu au Collège Manitou », loc. cit., 93.

118. George Miller, cité dans ibid. 


\section{ÉVEIL POLITIQUE ET LEADERSHIP}

Dans le but de compléter l'adaptation curriculaire proposée par le programme de sciences sociales, le cadre professoral qui était composé, en 1975, d'une douzaine d'enseignants autochtones (sur 23) ${ }^{119}$, était encouragé à enrichir ses contenus culturels ${ }^{120}$. Ghislain Picard, actuel chef de l'Assemblée des Premières Nations du Québec et du Labrador, souligne que les étudiants étaient eux-mêmes invités à proposer l'étude de notions qu'ils jugeaient utiles à leur propre développement, voire à l'actualisation des cultures autochtones: «On nous invitait en quelque sorte à développer ce que pouvait être notre propre vision de la philosophie autochtone, de la philosophie indienne ${ }^{121}$... Le projet Manitou visait à construire un pont entre les concepts traditionnels autochtones et les technologies du monde moderne dans une perspective de continuité culturelle ${ }^{122}$. La présence de l'imprimerie sur le campus favorisait la production de matériel pédagogique et didactique pour les différents programmes de La Macaza, notamment sous la bannière de la Thunderbird Press ${ }^{123}$. La concertation occasionnée au sein de Manitou permit à une génération de se rencontrer, de se découvrir et d'échanger sur des problématiques communes dans le but d'envisager des amorces de solutions à travers l'acquisition de connaissances jugées pertinentes sur le plan individuel et collectif. Comme l'explique Lise Bastien :

Toute la différence était là; on parlait de nous, de notre quotidien. Toutes les études de cas, c'étaient des cas de nos communautés. La littérature, les manuels; tout était axé sur les Premières Nations, sur les Autochtones [...]. Nous avions enfin l'occasion d'échanger sur des enjeux [...] ça a été super intéressant; les enjeux communs et les enjeux différents ${ }^{124}$.

Cette adaptation culturelle fut aussi identifiée comme un facteur de réussite par Kilfoil qui définissait, en 1979, la spécificité de Manitou en ces termes:

119. J. E. Bernard, Report of..., op. cit.

120. Entrevue avec Lise Bastien, le 18 août 2014; Gilles Allard et Ernie Benedict, dans M. Vincelette et L. Carter, «Vu et entendu au Collège Manitou », loc. cit., 89-89, 90-91; D. Cisneros, "Les lieux sauvages », loc. cit.

121. Ghislain Picard, cité dans R. Sioui Labelle, «L'éveil du pouvoir», loc. cit.

122. D. P. Foley, «L'éducation des minorités», loc. cit., 35.

123. Momik Sioui, cité dans M. Vincelette et L. Carter, "Vu et entendu au Collège Manitou», loc. cit., 89.

124. Entrevue avec Lise Bastien, le 18 août 2014. 
What made these subjects different from those offered in "White» CEGEPs is not only the fact that they were taught largely by native people, in a personal and informal environment, but also that course content was geared toward native cultures and the environment of the reservation [...] Thus, the relevance of the curriculum, the flexibility of the admission requirements, the basic upgrading courses offered, the relaxed and personal atmosphere of a small educational system and the predominance of native teachers and administrators contributed to making Manitou a unique learning environment ${ }^{125}$.

C'est d'ailleurs en matière d'éveil ${ }^{126}$ que de nombreux auteurs et leaders autochtones ayant étudié à Manitou décrivent leur expérience :

Le collège de La Macaza s'inscrivait directement dans notre intention d'offrir des cours en leadership pour éventuellement convaincre nos communautés qu'il fallait se prendre en main (Aurélien Gill) ${ }^{127}$.

La Macaza c'était le centre névralgique de tout ce qui se passait au niveau politique. Il y a plein d'informations qui nous étaient envoyées et qui ont réveillé ma conscience à moi (Ghislain Picard) ${ }^{128}$.

Le collège Manitou a été marquant pour la plupart des étudiants. Ça a certainement été l'éveil pour moi (Lise Bastien) ${ }^{129}$.

Si le régime des pensionnats indiens peut avoir indirectement contribué à l'essor d'une nouvelle élite intellectuelle et politique au sein des Premières Nations ${ }^{130}$, l'éducation visant le renforcement identitaire plutôt que l'acculturation forcée aura pour sa part permis d'assainir, d'ancrer, voire de magnifier la création d'un leadership autochtone à travers le Canada.

\section{LA FIN D'UN CYCLE}

En octobre 1975 fut déposé le rapport du comité de révision du projet Manitou formé de représentants du MAINC, de l'AIC et du Collège Manitou et coordonné par J. E. Bernard, du Service d'assistance canadien aux organismes ${ }^{131}$. L'initiative visait à évaluer les activités du Collège Manitou et à formuler des recommandations pour répondre aux difficultés

125. C. Kilfoil, Education and Identity Change..., op. cit.

126. A. Janin, «Le Collège Manitou (1973-1976)... », loc. cit.

127. R. Sioui Labelle, "L'éveil du pouvoir», loc. cit.

128. Ibid.

129. Ibid.

130. J. R. Miller, Shingwauk's Vision. A History of Native Residential Schools (Toronto, University of Toronto Press, 1996); M. P. Bousquet, "A Generation in Politics... », loc. cit.; G. Ottawa, Les pensionnats indiens au Québec..., loc. cit.

131. J. E. Bernard, op. cit. 
budgétaires qui se faisaient grandissantes. À la lumière du rapport, le comité se prononça en faveur du maintien du projet Manitou à La Macaza, soulignant le caractère innovant du collège et les prouesses administratives des dernières années ${ }^{132}$. Il évalua ses besoins financiers à 1,9 million, soit plus du double du montant de la subvention fédérale de $865000 \$$. Soulignant que plus de $80 \%$ du budget était associé à l'entretien et à la maintenance des infrastructures vieillissantes du campus, on recommanda notamment une indexation substantielle de la subvention du MAINC, un financement indépendant des installations physiques et matérielles de la Macaza ainsi que l'intégration de nouvelles sources de revenus ${ }^{133}$.

Quelques mois plus tard, à la suite de l'adoption de la Convention de la Baie-James et du Nord québécois en 1975, la nation inuite se retira du projet Manitou $^{134}$. La précarité du collège se trouvait également aggravée par la hausse du prix du pétrole décrétée par l'OPEP - qui augmenta considérablement les frais de chauffage des installations ${ }^{135}$ - et par la mauvaise presse dont le collège faisait l'objet à travers le Québec et les Maritimes ${ }^{136}$. Celle-ci était notamment alimentée par des incidents disciplinaires et des comportements subversifs d'abord rapportés dans les communautés par des étudiants, puis par les médias ${ }^{137}$. Or, aux dires plusieurs employés et étudiants, les problèmes évoqués ne concernaient que certains groupes ciblés sur le campus de La Macaza ${ }^{138}$ et la consommation d'alcool et de drogue y était somme toute comparable à celle rencontrée dans d'autres communautés ou, même, dans d'autres cégeps québécois ${ }^{139}$. Le climat anxiogène ne tarda pourtant pas à inquiéter les familles et les communautés participantes ${ }^{140}$. Certains chefs commencèrent à se montrer hésitants quant à l'octroi de leurs fonds d'éducation culturelle au projet Manitou ${ }^{141}$.

À l'hiver 1975, le gouvernement annonça la fin de la formule de financement quinquennal des centres d'éducation culturelle pour le 31 mars à

132. Ibid.

133. Ibid.

134. Entrevue avec Jacques Kurtness, le 20 décembre 2015. L’investissement du NQIA représentait environ 98735 \$ du budget annuel de Manitou, BAC, RG10-C-IV-9, 1996-97/978, 23, $28,30$.

135. Ibid. ; B. Stonechild, The New Buffalo..., op. cit., 58-61.

136. C. Kilfoil, Education and Identity Change..., op. cit., 28.

137. Ibid.; M. Vincelette et L. Carter, "Vu et entendu au Collège Manitou », loc. cit., 96; B. Stonechild, The New Buffalo..., op. cit., 60.

138. E. Benedict, cité dans M. Vincelette et L. Carter, "Vu et entendu au Collège Manitou », loc. cit. ;

C. Kilfoil, Education and Identity Change..., op. cit.

139. Ibid.

140. Ibid. ; BAC, RG10-C-IV-9, 1996-97/978, 12.

141. C. Kilfoil, Education and Identity Change..., op. cit.; Entrevue avec Jacques Kurtness, le 20 décembre 2015 . 
venir $^{142}$. Selon Stonechild, le Collège Manitou constituait le projet le plus ambitieux parmi les centres existants, fort de son accréditation provinciale $^{143}$. Le MAINC accorda une extension de deux mois au Collège Manitou, soit jusqu'à la fin mai 1976, le temps que les étudiants inscrits puissent compléter la session en cours ${ }^{144}$. La nouvelle de la réception d'un communiqué télex annonçant la fermeture éventuelle de Manitou ne tarda pas à se répandre sur le campus de La Macaza ${ }^{145}$.

Un rapport spécial fut déposé au MAINC le 28 janvier 1976 par la directrice académique, le contrôleur et le directeur général de Manitou pour désamorcer les nombreuses critiques essuyées par le collège et émettre des pistes de solution visant à assurer sa survie économique ${ }^{146}$. Le document s'employait à faire valoir le caractère unique du Collège Manitou, insistant sur le fait que ses services, jugés trop onéreux par le MAINC, desservaient une population composée entre autres de plus de 120 étudiants collégiaux ${ }^{147}$, de 160 à 180 étudiants du programme de formation des maîtres, d'une douzaine d'étudiants du programme de techniques d'amérindisation et de leurs familles respectives. Au cours de l'été 1975 seulement, 520 personnes y avaient séjourné ${ }^{148}$. Le campus de La Macaza hébergeait notamment les programmes de sciences sociales et d'arts-communication dispensés dans les deux langues d'instruction, le programme de formation des maitres de l'UQAC, le programme d'enseignement des langues autochtones, le programme articulé autour de la production de matériel pédagogique pour les écoles primaires et secondaires du Québec, les cours d'écriture syllabique du MAINC ainsi que différents autres séminaires thématiques, services de soutien aux communautés. Le rapport défendait ainsi le maintien de la formation postsecondaire autochtone à La Macaza, insistant sur le manque d'efficacité des initiatives tentées en contextes allochtones et sur les coûts additionnels à prévoir en cas de déménagement dans d'autres installations tel que le pensionnat de La Tuque ou celui de Pointe-Bleue. Les auteurs en

142. B. Stonechild, The New Buffalo..., op. cit.; BAC, RG10-C-IV-9, 1996-97/978, 23.

143. Ibid.

144. BAC, RG10-C-IV-9, 1996-97/978, 23.

145. Donna Augustine, dans Students of Manitou, Hear Our Words (La Macaza, Thunderbird Press, 1976).

146. V. Bourque et al., Special Report on Manitour Community College..., op. cit.

147. Selon le rapport du comité de révision, 128 étudiants collégiaux, soit 86 anglophones et 42 francophones, fréquentaient le collège Manitou au mois de septembre 1975. En fonction du niveau de complétion de leur parcours secondaire et de leurs résultats à des tests diagnostiques, ils étaient référés au programme collégial ou pré-collégial (préparatoire). Les étudiants âgés de plus de vingt ans étaient en majorité; ils étaient admis comme étudiants adultes en fonction de leur expérience de travail. J. E. Bernard, Review of..., op. cit.

148. Ibid., 8. 
appelaient à un financement récurrent du projet Manitou en tant qu' «institution éducative autochtone viable $\mathrm{e}^{149}$ " soutenue par les peuples autochtones, porteuse du projet de maîtrise indienne de l'éducation indienne et appelée à continuer de se développer ${ }^{150}$. On demandait ultimement que le MAINC accepte de repousser, au moins d'une année, la fin du financement prévu, le temps que soient étudiées les recommandations du rapport du comité de révision.

À l'annonce d'une fin imminente, un groupe d'étudiants algonquins, malécites, métis, micmacs, mohawks, naskapis, ojibwés et dénés, secondé par le professeur Don Whiteside et l'équipe étudiante de l'imprimerie Thunderbird Press, rédigea le manifeste illustré "Hear Our Words ${ }^{151}$ ", adressé au ministre des Affaires indiennes et du Nord Canada de l'époque, Judd Buchanan. Parmi les nombreux textes qui composent le livret de 47 pages, on pouvait lire les extraits suivants:

The government [at that time] voiced the opinion that in order for Canada to realize its maximum potential, the native peoples would have to be allowed to develop their cultural heritage [...]. The school can be thought of in terms of growing a tree. You start by planting the seed, and the seed is nurtured and cared for until it becomes a seeding, and then a sapling. [...] I for one, and many others of us at Manitou College, want the opportunity to grow in a way which will benefit our people and in so doing, benefit society at large (Ross L. Montour) ${ }^{152}$.

The education of Native people in Canada has been an embarrassment to the Canadian people. Since the days of Confederation, the Canadian government has had a program of assimilation to "help" the Native people fit into "their" mode of living [...]. Since its conception, Manitou College has made more advances in the education of Native people than the Canadian government has made in 109 years (Art Dedam) ${ }^{153}$.

Plusieurs lettres d’appui au projet Manitou furent envoyées par différentes instances autochtones et non autochtones au ministre Buchanan et au premier ministre Trudeau. Parmi les signataires se trouvaient les représentants de vingt et une réserves du Québec, de même que le Dr. George D. Spindler, professeur à la Stanford University et pionner de l'anthropologie de l'éducation, et les professeurs du département d'anthropologie de l'Université McGill ${ }^{154}$.

\footnotetext{
149. Ibid., 9, traduction libre.

150. Ibid., 9-10.

151. Students of Manitou, Hear Our Words, op. cit.

152. Ibid., 27-28.

153. Art Dedam dans ibid., 13.

154. BAC, RG10-C-IV-9, 1996-97/978, 12, 18.
} 
Au printemps 1976, soit avant même que soit publié le manifeste étudiant $^{155}$, le gouvernement fédéral accepta de reporter la fin du financement jusqu'en mai $1977^{156}$. Le Collège Manitou se vit octroyer une subvention spéciale de 707384 \$ pour boucler un budget annuel évalué à 1,3 million par la direction ${ }^{157}$. L'effritement des revenus aggravé par l'inflation ${ }^{158}$ fit en sorte que le Collège s'avéra entre autres incapable d'indexer les salaires de son personnel en dépit des demandes formulées ${ }^{159}$.

Empêtré dans une situation difficile, George Miller démissionna de son poste de directeur dans les semaines suivantes pour retourner à Stanford entamer des études doctorales ${ }^{160}$. C'est donc dans ce climat d'insécurité et de tensions latentes que le psychologue innu, Jacques Kurtness, âgé d’à peine trente ans, fut appelé à devenir le troisième et dernier directeur du collège par l'adoption d'une résolution du conseil d'administration votée le 9 mai $1976^{161}$. Au cours de la même rencontre, la directrice académique, Velma Bourque, présenta sa lettre de démission pour se réintégrer son poste de coordonnatrice du curriculum régional du MAINC ${ }^{162}$.

La nouvelle direction francophone, également amputée de son directeur adjoint (Stonechild) et de son contrôleur Dione (remplacé par Hinds), entra en fonction le $1^{\mathrm{er}}$ juillet. Elle se démarqua par une approche diamétralement différente des précédentes ${ }^{163}$. Tentant tant bien que mal de maîtriser certains problèmes partiellement attribuables à la consommation excessive d'alcool de certains groupes étudiants et non étudiants ${ }^{164}$, Kurtness sollicita la collaboration d'instances extérieures sur le campus de La Macaza, dont le ministère de la Santé et la Sûreté du Québec ${ }^{165}$. Ces interventions constituèrent une première dans l'histoire du collège et

155. Whiteside dans ibid., v; D. P. Foley, «L'éducation des minorités», 37; Entrevue avec Jacques Kurtness, le 20 décembre 2015.

156. Anonyme, «Un sursis d'un an au collège Manitou», Le Devoir, 18 mars 1976; BAC, RG10-C-IV-9, 1996-97/978, 12.

157. Le collège Manitou recevait également des revenus d'appoint (MEQ, revenus internes, Bureau régional du ministère des Affaires indiennes, ministère des Affaires culturelles, etc.) mais la somme de ceux-ci s'avérait en deçà des prévisions du budget d'austérité formulé par le contrôleur Dionne pour l’année 1976-1977. BAC, RG10-C-IV-9, 1996-97/978, 23.

158. BAC, RG10-C-IV-9, 1996-97/978, 28.

159. BAC, RG10-C-IV-9, 1996-97/978, 22.

160. Entrevue avec Jacques Kurtness, le 20 décembre 2015; V. Bourque et al., Special Report on Manitou Community College..., op. cit. ; BAC, RG10-C-IV-9, 1996-97/978, 12.

161. BAC, RG10-C-IV-9, 1996-97/978

162. BAC, RG10-C-IV-9, 1996-97/978, 22.

163. Entrevue avec Jacques Kurtness, le 20 décembre 2015 ; D. P. Foley, «L'éducation des minorités», loc. cit.

164. BAC, RG10-C-IV-9, 1996-97/978, 18.

165. Entrevue avec Jacques Kurtness, le 20 décembre 2015 ; BAC, RG10-C-IV-9, 1996-97/978, 20. 
furent hautement contestées ${ }^{166}$. Plusieurs exprimèrent leur mécontentement, accusant le nouveau directeur de prioriser une gestion verticale et strictement administrative, empêchant tous canaux de communication avec la population de Manitou ${ }^{167}$. Il en résulta une véritable guerre ouverte opposant l'administration du collège à certains groupes étudiants et employés ${ }^{168}$. Le Conseil des étudiants déclara entre autres la nomination de Kurtness comme illégitime et tenta d'obtenir sa démission ${ }^{169}$.

Dans la foulée de ces événements troubles, l'«hémorragie ${ }^{170}$ » budgétaire du collège continua à s'aggraver et finit par atteindre un paroxysme ${ }^{171}$. Le conseil d'administration du Collège Manitou se rencontra ainsi en présence de représentants du MAINC à Montréal le 4 décembre 1976 pour discuter de la viabilité d'un «budget d'austérité ${ }^{172}$ ». En cours de rencontre, le nouveau contrôleur du collège, $M$. Hinds, suivi du secrétaire exécutif du ministère, M. Gideon, livrèrent un aperçu de l'ampleur de la crise financière que traversait Manitou. Ce dernier invita le conseil à se questionner sur la possibilité de poursuivre les activités éducatives jusqu'au printemps 1977 considérant les dettes accumulées, le gel du compte de banque de l'institution et le manque de liquidité à prévoir ${ }^{173}$. À la suite d'échanges, il fut décidé, en dépit de la position des représentants étudiants et des professeurs, de suspendre les activités du projet Manitou jusqu'à nouvel ordre ${ }^{174}$ :

Chief Andrew Delisle, speaking on behalf of the Indians of Quebec Association, stated, "The chiefs expressed their concern that the [cultural education] monies stay at the Band level» to be spent on local initiatives. A resolution was passed "that the activities at the present site of the College be temporarily suspended; the students be reoriented towards other institutions for January 1977", and that a Management Committee be set up to study new structures. The motion was carried with five in favour and three abstentions ${ }^{175}$.

Les employés, les étudiants et le personnel du Collège Manitou furent avisés par écrit de la fermeture du Collège Manitou pour le 17 décembre de

166. Ibid.; D. P. Foley, «L'éducation des minorités», loc. cit.

167. Ibid.; C. Kilfoil, Education and Identity Change..., op. cit.

168. Ibid.

169. Ibid.

170. Entrevue avec J. Kurtness, le 20 décembre 2015.

171. B. Stonechild, The New Buffalo..., op. cit.

172. BAC, RG10-C-IV-9, 1996-97/978, 28, 30.

173. BAC, RG10-C-IV-9, 1996-97/978, 30.

174. Entrevue avec Jacques Kurtness, le 20 décembre 2015 ; BAC, RG10-C-IV-9, 1996-97/978, 17, 30.

175. B. Stonechild, The New Buffalo..., op. cit.; BAC, RG10-C-IV-9, 1996-97/978, 30. 
la même année et de leur obligation d'avoir quitté le campus de La Macaza dans un délai de deux jours après cette date ${ }^{176}$. Il en résulta d'un vif mouvement de contestation écourté par l'intervention de la Sûreté du Québec et l'application de mesures incitatives pour accélérer l'évacuation de la base ${ }^{177}$. La population étudiante était alors d'environ 150 étudiants ${ }^{178}$.

Comme en témoignera Cisneros, la fermeture subite de Manitou eut un effet dévastateur sur les étudiants, tout comme sur les enseignants impliqués dans la lutte pour sa survie:

J'ai continué à La Macaza mon labeur de loup de corbeau, ramassant les cadavres de la forêt, les mangeant, tannant leurs peaux, momifiant, nettoyant les ossements, ramassant des écorces et des racines, faisant des expériences avec des branches et des mousses, des cornes et des sabots. Mais j'étais fâché. Les nouvelles œuvres que je faisais étaient très différentes de celles de la période du Collège Manitou. La mort de ce dernier m’avait laissé une cicatrice ${ }^{179}$.

Selon Beaudoin, ce coup de théâtre fut en fait le résultat de mesures de fragilisation économique du projet Manitou par le MAINC:

Est-ce dire que l'action dysfonctionnelle des hauts fonctionnaires faisait place à plus de cohérence envers les intérêts supérieurs du régime ou si tout simplement ces derniers espéraient que l'ouvrage difficile serait exécuté par les Amérindiens eux-mêmes suite aux difficultés financières et aux limites opérationnelles d'un tel campus ${ }^{180}$ ?

En marge des arguments d'ordre financier et de la crise interne qui furent évoqués pour justifier la fermeture hâtive du collège de La Macaza, des voix dissidentes postulèrent - et postulent encore aujourd'hui - que l'éveil politique et le foisonnement d'un nouveau militantisme autochtone auraient inquiété les autorités au pouvoir ${ }^{181}$. Selon Kurtness, ce militantisme, associé à la montée du mouvement Red Power, aurait fait craindre à la GRC une menace terroriste autochtone alors que Montréal s'apprêtait à recevoir les Jeux olympiques ${ }^{182}$. Avec le recul, le troisième et dernier

176. BAC, RG10-C-IV-9, 1996-97/978, 17.

177. Entrevue avec Jacques Kurtness, le 20 décembre 2015 ; BAC, RG10-C-IV-9, 1996-97/978, 17.

178. C. Kilfoil, Education and Identity Change..., op. cit.

179. D. Cisneros, «Les lieux sauvages», op. cit., 21.

180. J. Beaudoin, «Pourquoi la Macaza», op. cit., 31.

181. Ibid. B. Stonechild, The New Buffalo..., op. cit.; Max Groslouis dans R. Sioui Labelle, «L'éveil du pouvoir", loc. cit.; Entrevue avec Lise Bastien, le 18 août 2014.

182. Entrevue avec Jacques Kurtness, le 20 décembre 2015. Voir également Jean Morisset, «Miroir indogène reflet eurogne», Recherches amérindiennes au Québec, 9, 4 (1980) et Rémi Savard dans R. Sioui Labelle, "L’éveil du pouvoir», loc. cit. 
directeur de Manitou dénonce également le caractère opportuniste du gouvernement fédéral dans la gestion financière du dossier de La Macaza :

Les Autochtones ont financé le fonctionnement et l'entretien de la base militaire de La Macaza le temps de trouver une autre vocation, à savoir un pénitencier à sécurité intermédiaire. Cette dernière vocation est perçue comme étant plus appropriée pour l'utilisation de la base [...], plus payante, plus acceptable $[\mathrm{ou}]$ moins dérangeante pour la population du coin et son député [de l'époque ${ }^{183}$.

La corporation du Manitou Community College ne fut dissoute qu'en 1996 , soit vingt ans après sa fermeture ${ }^{184}$.

\section{CONCLUSION}

En somme, plus d'une centaine d'étudiants autochtones auront obtenu un diplôme du Collège Manitou entre 1973 et $1976^{185}$. Un nombre équivalent d'étudiants autochtones se serait vu refuser l'accès au collège par le MAINC, faute de financement ${ }^{186}$.

La fermeture du Collège Manitou s'inscrit dans un tableau multifactoriel. Or, pour Gail Valaskakis, membre du conseil fondateur du Collège, le manque de vision des décideurs publics, en l'occurrence du MAINC, et leur incapacité à évaluer les retombées à long terme, auraient eu raison $\mathrm{du}$ premier projet postsecondaire autochtone du Québec ${ }^{187}$. Selon Cadieux, l'institut collégial aurait été victime de son époque, ouvrant 10 ans avant son temps ${ }^{188}$.

Près de trente-cinq années après la fermeture du Collège Manitou ouvrait l'Institution Kiuna dans la communauté abénaquise d'Odanak. Elle constitue, depuis 2011, la seule institution postsecondaire par et pour les étudiants des Premières Nations au Québec. Le projet, porté par Lise Bastien, ancienne étudiante de Manitou devenue directrice du CEPN, aura nécessité plus de dix années de préparation et de concertation avec l'Assemblée des Premières Nations du Québec et du Labrador, ses communautés et son chef Ghislain Picard (également ancien étudiant de Manitou). Tout comme les centres d'éducation culturelle qui l'ont précédée, l'Institution Kiuna souhaite offrir un cadre culturellement sécuritaire et adapté aux

\footnotetext{
183. Entrevue avec Jacques Kurtness, le 20 décembre 2015.

184. Federation Corporation Information, Canada-053568-1.

185. C. Kilfoil, Education and Identity Change..., op. cit.

186. Bourque et al., Special Report on Manitou Community College, op. cit.

187. Dans B. Stonechild, The New Buffalo..., op. cit.

188. Entrevue avec Rémi Cadieux, le 5 janvier 2015.
} 
besoins des étudiants des Premières Nations. Elle dispense le programme collégial de sciences humaines - Premières Nations ainsi que différents programmes courts élaborés en fonction des besoins des communautés ${ }^{189}$. On y retrouve plusieurs conditions favorables à la réussite instaurées par le Collège Manitou: adaptation culturelle des contenus, renforcement de l'esprit communautaire, création d'une identification panindienne inclusive, enseignement bilingue réparti entre deux cohortes linguistiques collégiales, présence d'enseignants et de personnel autochtones, affiliation aux cégeps provinciaux (Cégep Abitibi-Témiscamingue et au Collège Dawson), reconnaissance ministérielle, approches visant le développement du leadership et de la fierté culturelle, certifications sur mesure, etc.

Tout comme le Collège Manitou, l'Institution Kiuna peut se targuer de très bons taux de rétention des étudiants, soit $85 \%$ en 2015. À l'hiver 2016, elle avait délivré plus de 28 diplômes d'études collégiales et prévoyait 12 nouveaux diplômés autochtones pour le printemps de la même année, en plus des autres cohortes techniques et professionnelles ${ }^{190}$. L'analyse des données recueillies auprès des étudiants de Kiuna a précédemment permis de confirmer que l'adaptation culturelle de programme et de services prodiguée par l'Institution - ou même le Collège Manitou - favorise indubitablement la persévérance scolaire, le renforcement de l'identité culturelle et donc, la réussite éducationnelle d'étudiants autochtones ${ }^{191}$. Forte des leçons tirées de l'expérience de Manitou et d'un contexte sociopolitique et économique relativement distinct, Kiuna se différencie néanmoins de son prédécesseur - identifié par Bastien comme «la genèse » ${ }^{192}$ - au niveau de son financement, de sa structure administrative, de ses installations, etc. Les petites et grandes victoires remportées au quotidien par le Collège Manitou, l'Institut Kiuna, ainsi que celles des Tribal Colleges and Universities, tendent néanmoins à renforcer l'idée que l'éducation est intrinsèquement et réciproquement liée à la culture.

La réactivation des mémoires du Collège Manitou veille à nous rappeler que dans le contexte politique et économique actuel, la mise en place et la survie d'initiatives éducationnelles autochtones dépendent notamment de la mise en place, par nos décideurs publics, de conditions de développement favorables. Au lendemain de l'adoption des appels à l'action de la

189. Voir E. Dufour, “Une école où tu apprends à être fier de ce que tu es”: L'Institution Kiuna et le programme sciences humaines-Premières Nations», loc. cit., 163-176.

190. Statistiques maison fournies par la direction de l'Institution Kiuna à l'auteure.

191. Ibid.; V. Bourque et al., Special Report on Manitour Community College..., op. cit.; Entrevue avec Lise Bastien, le 18 août 2014.

192. Entrevue avec Lise Bastien, le 18 août 2014. 
Commission de vérité et réconciliation par le gouvernement de Justin Trudeau ${ }^{193}$, ces initiatives constituent des investissements à la fois prometteurs et essentiels aux mouvements de revitalisation des langues et des cultures autochtones. Elles s'inscrivent en outre dans un processus d'autonomisation et de prise en charge de l'éducation autochtone, amorcé par les principaux intéressés depuis plusieurs décennies.

193. Justin Trudeau, premier ministre du Canada, Rapport final de la Commission de vérité et réconciliation du Canada, www.pm.gc.ca/fra/nouvelles/2015/12/15/rapport-final-de-la-commissionde-verite-et-reconciliation-du-canada (consulté en janvier 2017). 